\title{
IMPORTANCIA DE LOS MAÍCES NATIVOS DE MÉXICO EN LA DIETA NACIONAL. UNA REVISIÓN INDISPENSABLE
}

\author{
IMPORTANCE OF MEXICAN MAIZE LANDRACES IN THE NATIONAL DIET. \\ AN ESSENTIAL REVIEW
}

\author{
Rocío Fernández Suárez ${ }^{1}$, Luis A. Morales Chávez ${ }^{3}$ y Amanda Gálvez Mariscal ${ }^{1,2 \star}$
}

\begin{abstract}
${ }^{1}$ Programa Universitario de Alimentos, Edificio de Programas Universitarios, Universidad Nacional Autónoma de México (UNAM), Ciudad Universitaria. Circuito de la Investigación Científica s/n. 04510, Distrito Federal, México. Tels. (55) 5622 5208, (55) 5616 6816. ${ }^{2}$ Facultad de Química, Edificio de Alimentos y Química Ambiental, Laboratorio 312, Conjunto E (UNAM), Ciudad Universitaria. Circuito de la Investigación Científica s/n. 04510, Coyoacán, D.F. Tel. (55) 5622 5305. ${ }^{3} \mathrm{Fa}-$ cultad de Estudios Superiores Cuautitlán, Campo 4, UNAM. km 2.5 Carr. Cuautitlán-Teoloyucan. 54714, San Sebastián Xhala, Cuautitlán Izcalli, Edo. de México.
\end{abstract}

${ }^{*}$ Autor para correspondencia (galvez@unam.mx)

\section{RESUMEN}

En México las numerosas variedades de maíces (Zea mays L.) nativos se utilizan para elaborar además de la tortilla, una enorme cantidad de preparaciones culinarias tradicionales, lo que hace del maíz uno de los elementos fundamentales de la cocina nacional. Estos maíces siguen siendo el sustento de miles de familias rurales mexicanas. Sin embargo, ante el proceso de globalización se ha adoptado un estilo "moderno" de alimentación que tiende a sustituir a los platillos tradicionales por alimentos procesados, no necesariamente basados en maíz. Este hecho, junto con otros factores como el abandono del campo, la pérdida de memoria biocultural y el cambio climático, hacen que peligre la existencia de los maíces nativos. Conservarlos es una tarea ineludible que requiere definir estrategias a corto plazo. En este trabajo se hace explícito el papel fundamental que actualmente desempeñan los maíces nativos en la dieta mexicana, pese a los cambios registrados en los patrones de consumo alimentario. También se enlistan las preparaciones culinarias tradicionales que tienen como base el maíz, con énfasis en la relación que existe entre usos especiales, razas nativas y las características fisicoquímicas de los granos. Se destaca la necesidad de potenciar la demanda de maíces nativos a partir de la revalorización de los usos tradicionales, así como del impulso de usos novedosos y prácticas alternativas que no han sido suficientemente explotadas. Todas estas acciones son parte de las estrategias para coadyuvar en la conservación in situ de los maíces nativos mexicanos..

Palabras clave: Zea mays, maíces nativos, usos culinarios, usos especiales, dieta mexicana.

\section{SUMMARY}

In México, numerous varieties of maize (Zea mays $\mathrm{L}$.) landraces are employed for preparation of a great amount of traditional culinary dishes in addition to tortillas. Maize is a fundamental element of the national cuisine. Additionally, native landraces are the livelihood of thousands of Mexican rural families. However, under globalization tendencies, "modern" eating habits are being adopted, substituting traditional dishes for processed foods, which are not necessarily maizebased. This fact, along with other factors such as biocultural memory loss, agricultural activities abandonment, or climate change endanger landrace existence. Conserving native maize varieties is an urgent task, which requires short-term strategies. This review describes the fundamental role maize landraces play in the Mexican diet, in spite of changes in food consumption patterns already registered in México. A number of traditional maize-based culinary preparations, emphasizing the relationship between special uses, races and the physicochemical characteristics of the grains, are described. This review promotes demand increments of native maize landraces based on reevaluation of traditional uses, as well as promotion of novel uses and alternative practices that have not been properly exploited. All these actions take part of the strategies for in situ conservation of Mexican maize landraces.

Index words: Zea mays, maize landraces, culinary uses, special uses, Mexican diet.

\section{INTRODUCCIÓN}

En México, centro de origen, domesticación y diversificación del maíz (Zea mays L.), existen 59 razas de acuerdo con la clasificación más reciente basada en características morfológicas e isoenzimáticas (Sánchez et al., 2000), que representan un significativo porcentaje de las 220 a 300 razas de maíz existentes en el continente americano (Kato et al., 2009). Esta diversidad es producto de milenarias prácticas agrícolas vinculadas al conocimiento tradicional de los pueblos indígenas de México, principales herederos, custodios y mejoradores del germoplasma nativo (Mera-Ovando y Mapes-Sánchez, 2009; Turrent et al., 2010; Toledo-Manzur y Barrera-Bassols, 2008). De hecho, el mejoramiento genético del maíz es una actividad que en México probablemente se remonta a más de 10 mil años (Miranda-Colín, 2000).

Desde el punto de vista alimentario, político, económico y social, el maíz es el cultivo más importante del país (SIAP, 2007). Basta con decir que el consumo per capita de maíz en México es aproximadamente 10 veces mayor que el de Estados Unidos de América (Serna-Saldívar y AmayaGuerra, 2008). Este cereal cubre poco más de la mitad de la superficie agrícola sembrada, con aproximadamente 7.5 millones de hectáreas (SIAP, 2011), principalmente en las zonas sub-húmeda tropical, templada húmeda y sub-húmeda (Mera-Ovando y Mapes-Sánchez, 2009). 
De la superficie total sembrada con maíz, la mayor parte (80\%) es de temporal o secano (SIAP, 2011), fundamentalmente a cargo de más de 2 millones de productores a pequeña escala, quienes lo siembran sobre todo para autoconsumo (Mera-Ovando y Mapes-Sánchez, 2009). Más de la mitad de la producción nacional de maíz proviene de este sistema (Turrent et al., 2012), el cual también es conocido como de subsistencia porque contribuye significativamente a la seguridad alimentaria de los estratos rurales más pobres (Turrent et al., 2012). Es aquí en donde los maíces nativos se seleccionan, producen, conservan, diversifican y domestican de acuerdo con las necesidades de las poblaciones locales (Turrent et al., 2010; Turrent et al., 2012).

Por su parte, los maíces mejorados (híbridos) son los que satisfacen en buena medida las necesidades de la agroindustria mexicana, y ocupan tan solo $20 \%$ de la superficie total sembrada con maíz (SIAP, 2011). Se producen principalmente bajo sistemas de riego en el noroeste de México, en donde se registra un uso notable de agroquímicos.

En términos de rendimiento, las variedades mejoradas han mostrado ser notablemente superiores a las nativas (Turrent et al., 2012), pero los pequeños productores suelen preferir sus variedades locales. Esto se debe a ciertas ventajas que se han identificado en las razas nativas, que en su mayoría se siembran en los terrenos edafo-climáticamente más limitativos (Turrent et al., 2012). De hecho, se han reportado razas que pueden sobrevivir donde las variedades mejoradas no tienen oportunidad (Vázquez-Carrillo et al., 2010). Entre las ventajas de estos maíces destacan las siguientes: mejor manejo del riesgo agrícola, adaptación a las condiciones climáticas locales, estabilidad a la variabilidad climática, costos más bajos de los insumos necesarios para su producción, y muy importante, aptitud para la elaboración de preparaciones culinarias tradicionales (GuillénPérez et al., 2002; Turiján-Altamirano et al., 2012; Turrent et al., 2012).

Por todo lo anterior, la protección, caracterización y conservación del germoplasma nativo, así como los saberes y conocimientos tradicionales asociados, son acciones impostergables, sobre todo ante las evidencias que existen de pérdida y extinción de algunas poblaciones (Lazos y Chauvet, 2011; Ortega-Paczka, 2003; Ortega-Corona et al., 2011 ), situación que podría agudizarse ante los efectos del cambio climático (Bellon et al., 2011).

Con respecto a la caracterización de las razas nativas, cabe mencionar que durante mucho tiempo la investigación se ha centrado en el rendimiento de materia seca del grano y otros rasgos agronómicos (Turrent et al., 2012). Sólo recientemente se han realizado estudios científicos sobre los aspectos de calidad que determinan los usos culinarios específicos, como aquí se revisa en una sección posterior. Las investigaciones en este campo, junto con estudios de cadenas de valor e implementación de políticas de Estado pertinentes, pueden contribuir notablemente a impulsar estratégicamente el uso de maíces nativos, y por tanto también a su conservación in situ (Hellin et al., 2010; Keleman y Hellin, 2009).

El presente artículo de revisión tiene por objetivo identificar los beneficios que los maíces nativos proporcionan a la población de México, con énfasis en la importancia que actualmente tienen en la culinaria nacional y en la dieta de los mexicanos. También se busca identificar usos novedosos y prácticas alternativas que hasta el momento no han sido suficientemente explotadas. Todo esto con el fin último de sustentar la necesitad de proteger el germoplasma nativo y garantizar su conservación in situ.

\section{EL MAÍZ, ELEMENTO FUNDAMENTAL DE LA DIETA MEXICANA}

La dieta de una población particular forma parte de la memoria colectiva, y no solo comprende la ingesta de alimentos sino también expresa relaciones socioeconómicas y hace patente actos profundamente cargados de simbolismo cultural (García-Urigüen, 2012). Es por estas razones que resulta indispensable definir con claridad el significado que ha tenido el maíz en la dieta de la población mexicana a lo largo de la historia y en la actualidad.

Existen testimonios culinarios del maíz (restos arqueológicos y manuscritos como el códice Florentino o el Mendocino) que permiten concluir que se trataba de uno de los componentes de la dieta mesoamericana desde el Preclásico Medio (1200-400 a.C.) (López, 2007; Taube, 1989). Sin embargo, diversos restos arqueológicos indican que otras plantas predominaron durante mucho tiempo en la dieta de los antiguos pobladores, y que el maíz fue ganando popularidad poco a poco (Ortega-Paczka, 2003). Algunos usos probables en la época prehispánica eran la producción de harinas, pinole y granos reventados con calor hasta que explotaran, en la forma que hoy conocemos como "palomita de maíz" (Mera-Ovando, 2009). Es posible que también se bebiera el jugo dulce de la caña del maíz y del teocintle (Mera-Ovando, 2009). Con respecto a la tortilla, hoy se sabe que ésta no era conocida al principio (Ortega-Paczka, 2003), aunque a la llegada de los españoles ya predominaba en la dieta mesoamericana. Hoy por hoy, la tortilla es considerada como la base de la supervivencia del pueblo mexicano desde hace más de 3500 años (Paredes-López et al., 2009). 
La riqueza de la gastronomía indígena basada en el maíz fue asentada en testimonios fehacientes de los conquistadores y cronistas, desde Hernán Cortés y Bernal Díaz del Castillo, hasta fray Bernardino de Sahagún quienes expresaron pruebas del alto grado de desarrollo cultural de los antiguos mexicanos, así como de la diversidad existente, ya en esos días, del maíz (Echeverría y Arroyo, 2000).

El mestizaje que se dio a raíz de la "conquista española" tuvo en la gastronomía una de sus principales manifestaciones, al enriquecer la dieta prehispánica con ingredientes de la cocina hispano/árabe, y viceversa. Sin embargo, la cocina indígena predominó en el "mestizaje alimentario", pues el maíz, que es la aportación más significativa, sigue siendo el ingrediente fundamental y una de las principales fuentes de energía en la dieta actual de México. En promedio, un mexicano recibe diariamente del maíz 1022 kcal y 26.3 g de proteína (FAOSTAT, 2009), lo que puede representar $50 \%$ de la ingesta diaria de una persona adulta, si se toma como base una dieta de 2000 kcal y 56 g de proteína (SernaSaldívar y Amaya-Guerra, 2008).

Además surgieron nuevos usos del maíz, como el cuitlacoche o huitlacoche, que es un "invento mexicano" reciente cuyo uso se ubica no hace más de cien años, aunque ya se encuentra arraigado en la culinaria nacional (Valadez et al., 2011). Se conoce como cuitlacoche a las agallas jóvenes generadas por el hongo basidiomiceto Ustilago maydis en mazorcas inmaduras o frutos en formación (Juárez et al., 2011). Aunque a nivel mundial es visto como una enfermedad, en México es considerado un valioso alimento con potencial nutracéutico (Beas et al., 2011; Juárez et al., 2011).

Los mexicanos heredamos una de las tradiciones culinarias más variadas y saludables que existen en el mundo actual. No obstante, ante el ímpetu de "modernización" y la fuerza económica de la industria alimentaria, poco a poco se ha adoptado una dieta que incorpora significativamente alimentos procesados, menos saludables y de mayor densidad energética, lo que conduce a un consumo más frecuente de grasas saturadas, azúcares y sal (Bourges-Rodríguez, 2004; Gálvez-Mariscal y Bourges-Rodríguez, 2012). Paralelamente, ha disminuido el consumo de platillos tradicionales basados en maíz y otros cultivos ricos en nutrimentos procedentes de la milpa (Gálvez-Mariscal y Bourges-Rodríguez, 2012). Esta transición alimentaria, además de menguar la salud de la población, pone en peligro la existencia de los maíces nativos y otros valiosos alimentos de la dieta tradicional mexicana

Los cambios en el patrón alimentario de la población mexicana ya se han reflejado en el consumo de uno de los alimentos más populares, la tortilla de maíz. De 1980 a la actualidad, el consumo de este producto ha disminui- do aproximadamente a la mitad, mientras que el consumo de pan de trigo (Triticum spp) ha mostrado una tendencia a la alza (García-Urigüen, 2012). Aún así, la tortilla sigue siendo uno de los alimentos predominantes en la dieta mexicana. Alrededor de $82 \%$ de los hogares incluyen a las tortillas en su dieta, y representa $6.4 \%$ del gasto total en alimentos, aunque la población de menores ingresos puede destinar más de $25 \%$ de su presupuesto alimentario en este producto (INEGI, 2010). De acuerdo con cálculos recientes del Consejo Nacional de Evaluación de la Política de Desarrollo Social, el consumo diario per capita de tortilla es de $155.4 \mathrm{~g}$ en las zonas urbanas y hasta $217.9 \mathrm{~g}$ en las zonas rurales (CONEVAL, 2012). La importancia de la tortilla en la dieta no es menor, pues se trata de una excelente fuente de calorías y calcio (Serna-Saldívar y Amaya-Guerra, 2008) que puede proporcionar de 32 a $62 \%$ de los requerimientos mínimos de hierro (Paredes-López et al., 2009)

\section{PREPARACIONES CULINARIAS TRADICIONALES Y USOS ESPECIALES}

La cocina tradicional mexicana, que tiene como base al maíz, es considerada Patrimonio Cultural Inmaterial de la Humanidad por la Organización de Naciones Unidas para la Educación, la Ciencia y la Cultura (UNESCO, 2010). Si hubiera alguna duda en cuanto al origen del maíz, bastaría con hacer un recuento del número de platillos que se preparan con este cereal, muchos de ellos desde tiempos remotos. El destacado antropólogo Eusebio Dávalos Hurtado decía que en México existen no menos de 700 formas de comer el maíz, afirmación que el "Recetario del Maíz" editado por el Consejo Nacional para las Culturas y las Artes (CONACULTA) sustenta y amplía considerablemente (Echeverría y Arroyo, 2000). En el Cuadro 1 se reportan algunas de las preparaciones culinarias más tradicionales. Es importante subrayar que la base de estos platillos son los maíces nativos y no los mejorados, los cuales no reúnen las propiedades y calidad necesaria, en la mayoría de los casos, para la preparación de platillos específicos (Ortega-Paczka, 2003) Cuadro 1.

En muchos casos se ha encontrado una correlación estricta entre la raza de maíz y el tipo de preparación culinaria. Por ejemplo, la raza Bolita es la idónea para elaborar la tortilla "tlayuda" y el "tejate", con la raza Cacahuacintle se prepara el pozole, la raza Harinoso de Ocho se prefiere para la elaboración de "coricos", la raza Bofo se usa para hacer "huacholes", y la raza Zapalote Chico es la ideal para elaborar el "totopo istmeño". Justamente estos son algunos de los "usos especiales" de las variedades nativas, que se definen como los usos culinarios específicos descritos para una raza en particular, y que pueden ser distintivos de una región o cultura determinada. 
Cuadro 1. Alimentos y preparaciones culinarias tradicionales preparados con maíz.

\begin{tabular}{ll}
\hline Tipo de preparación & Ejemplos de alimentos y preparaciones culinarias \\
\hline & Tortillas, totopos istmeños, tlayudas, chilaquiles, enchiladas, enfrijoladas, entomatadas, tacos, tostadas, \\
Tortillas, antojitos, & quesadillas, garapaches, panuchos, papatzules, enjococadas, chopas de perico, chalupas, gorditas, molo- \\
botanas y similares & tes, peneques, sopes, tlacoyos o tlatloyos, salbutes, palomitas, totopos, nachos, frituras, otros.
\end{tabular}

Elotes y sopas Elote, cuitlacoche, esquites, pozoles y menudos, chacales, chicales, huachales, chochoyotes, sopas, otros.

Tamal. De elote y de nixtamal. Dulces y salados. Con y sin relleno. De cazuela. Joroch. Nacatamales, kehil hua, buulil hua, zacahuil, pibipollo, tobi holoch, colados, chanchamitos, pictes de elote, uchepos,

Tamales y similares corundas, agrios, colados, con frijoles, de garbanzo, de cacahuate, de tortilla, de tismiche, de ceniza, de chaya, de juacane, de chipilín, de frutas (piña, coco, naranja, almendra, avellanas, ciruela pasa guayaba), otros.

Pinole, tascalate, “alfajores”, batarete yaqui, ponteduro, burritos de maíz, manjar de maíz azul, "maría

Pinoles, dulces y repostería

Atoles

Bebidas gorda", melcocha, memenshas, tepopoztes, pemoles, totopos de huetamo, boronitas, coricos, buñuelos, gorditas tradicionales, de cuajada, de piloncillo, de maíz cacahuacintle, gondoches de pabellón, galletas de Zacazonapan, pan de maíz, pan de elote, tortas de maíz, turuletes de maíz, tlaxcales, toqueras de elote, otros.

Atole: blanco, nuevo, agrio, usua, champurrado, chileatole, cuatole, nicuatole, malarrabia, tanchucuá, nixteme, de pinole, de frijol, de cacahuate, de avellana, de frutas, de chiles, de pepita, de aguamiel, de coyol, de grano, común de sabores varios (chocolate, vainilla, etc.), otros.

Pozol, chorote, tesgüino (teshuino o tejuino), yorique, chicha, chilote, elisquiate, menjengue, piznate, otros.

Elaboración propia a partir de lo reportado en CONABIO (2011) y Echeverría y Arroyo (2000). Los ejemplos resaltados en negritas corresponden a aquellos usos que fueron reportados en la más reciente colecta nacional de maíces nativos.

La más reciente colecta nacional de maíces nativos (Proyecto CONABIO, 2011; Informe $)^{1}$ sustentó la vigencia que tienen los usos culinarios de diversas razas nativas. Un minucioso análisis de Lazos y Chauvet (2011) hecho a partir de la colecta mencionada, puso de manifiesto la importancia que los usos culinarios tienen en la selección de semillas. El agricultor mexicano tradicional selecciona las semillas más aptas con base en su resistencia a enfermedades, productividad y las características que se desean en los productos finales (sabor, color, textura, facilidad de cocción, entre otras) (Lazos y Chauvet, 2011). Por tanto, no es casual que predominen las razas de grano dentado y con dureza intermedia, que son las más aptas para la elaboración de tortillas (Ortega-Paczka, 2003).

En el Cuadro 2 se presentan los usos más comunes en la actualidad y las razas nativas asociadas.

Es importante resaltar que hay un gran número de razas asociadas con la elaboración de tortilla, y por otro lado pueden identificarse varios usos para una misma raza. De hecho, Narváez-González et al. (2007) reportaron que a di-

\footnotetext{
Proyecto Global: "Recopilación, generación, actualización y análisis de información acerca de la diversidad genética de maíces y sus parientes silvestres en México (CONABIO, 2011).
}

ferencia de las razas de Centroamérica y el Caribe, las razas de México y Sudamérica tienen una amplia variedad de usos, e identificaron al menos tres diferentes. Por ejemplo, la raza Cacahuacintle que corresponde al maíz pozolero por excelencia, también destaca por su calidad elotera y por su uso para elaborar galletas tradicionales en el centro del país. De acuerdo con lo reportado en el Cuadro 2, con esta raza también se pueden elaborar tortillas, harinas, atoles y pinoles.

\section{OPERACIONES CULINARIAS}

La culinaria tradicional basada en el maíz ha requerido del desarrollo de sofisticadas operaciones y técnicas culinarias, tanto para la preparación de alimentos como para su conservación: nixtamalización, cocción al vapor y en horno subterráneo, fermentación, molienda para la preparación de harinas, reventado, deshidratado, salado, ahumado, asado y otras.

Cabe destacar que los diferentes procesamientos pueden contribuir a incrementar el valor nutritivo de los alimentos preparados. Un ejemplo es el pozol que se elabora a partir de masa de nixtamal sometida a una fermentación láctica, con lo que se obtiene una bebida rica en probióticos 
Cuadro 2. Usos comunes y razas nativas asociadas.

\begin{tabular}{ll}
\hline Uso & \multicolumn{1}{c}{ Razas } \\
\hline \multirow{2}{*}{$\begin{array}{l}\text { Tortillas y } \\
\text { relacionados }\end{array}$} & Comiteco, Conejo, Cónico, Coscomatepec, Cristalino de Chihuahua, Dulcillo del Noroeste, Elotero de \\
& Sinaloa, Elotes Cónicos, Elotes Occidentales, Gordo, Harinoso de Ocho, Jala, Mushito, Nal-Tel de Altura, \\
& Olotillo, Olotón, Onaveño, Palomero de Chihuahua, Palomero Toluqueño, Pepitilla, Reventador, Tabloncillo, \\
& Tepecintle, Tuxpeño, Tuxpeño Norteño, Vandeño, Zapalote Chico (totopo), Zapalote Grande.
\end{tabular}

Ancho, Apachito, Blando de Sonora, Bofo, Cacahuacintle, Chapalote, Comiteco, Complejo Serrano de Elotes Jalisco, Conejo, Cónico, Coscomatepec, Dulce, Dulcillo del Noroeste, Elotero de Sinaloa, Elotes Cónicos, Elotes Occidentales, Gordo, Harinoso de Ocho, Jala, Nal-Tel, Olotón, Pepitilla, Tabloncillo, Tabloncillo Perla, Tepecintle, Tuxpeño, Zapalote Grande.

Galletas y dulces

Blando de Sonora (coricos), Bofo (galletas), Cacahuacintle (galletas), Chalqueño (burritos), Elotes Occidentales (chicales), Gordo (galletas, harinillas), Harinoso de Ocho (coricos), Reventador, Tepecintle.

Palomitas

Apachito, Arrocillo Amarillo, Chapalote, Nal-Tel, Palomero de Chihuahua, Palomero Toluqueño, Reventador.

Apachito, Azul, Celaya, Chapalote, Comiteco, Complejo Serrano de Jalisco, Cónico, Cónico Norteño,

Botanas Coscomatepec, Cristalino de Chihuahua, Dulce de Jalisco, Dzit Bacal, Elotes Occidentales, Jala, Onaveño, Tablilla de Ocho, Tabloncillo, Tabloncillo Perla, Tehua, Tuxpeño, Tuxpeño Norteño, Vandeño, Zamorano Amarillo, Zapalote Chico, Zapalote Grande.

Pozoles, sopas y Ancho, Blando de Sonora, Bofo, Bolita, Cacahuacintle, Chalqueño, Cónico Norteño, Dulce, Dulcillo del menudos Noroeste, Elotes Occidentales, Gordo, Harinoso de Ocho, Jala, Mushito, Tabloncillo, Tuxpeño, Vandeño.

Apachito, Arrocillo, Azul, Blando de Sonora, Bofo, Cacahuacintle, Chalqueño, Comiteco, Conejo,

Atoles Coscomatepec, Cristalino de Chihuahua, Elotes Cónicos, Elotes Occidentales, Harinoso de Ocho, Mushito, Nal-Tel, Olotón, Pepitilla, Tehua, Tepecintle, Tuxpeño, Tuxpeño Norteño, Zapalote Grande

Pinoles

Apachito, Azul, Bofo, Cacahuacintle, Chalqueño (rojo-amarillo), Chapalote, Cónico, Dulce, Dulcillo del Noroeste, Elotes Cónicos, Gordo, Jala, Onaveño, Reventador, Tabloncillo, Tablilla de Ocho.

Harinas

Cacahuacintle, Celaya, Elotes Cónicos, Gordo, Harinoso de Ocho, Mushito, Olotón, Vandeño.

Bebidas

Apachito (tejuino, tesgüino), Azul (tesgüino), Bofo (huajatole, tesgüino), Bolita (tejate), Comiteco (pozol), Nal- Tel (pozol), Olotón (pozol), Tabloncillo (piznate), Tablilla de Ocho (tesgüino), Tepecintle (pozol), Tuxpeño (pozol), Zapalote Grande (pozol).

Elaboración propia con información obtenida de CONABIO (2011) y SINAREFI (2012).

(Wacher et al., 1993) y con mayor contenido de aminoácidos (Cravioto et al., 1955).

La nixtamalización del maíz, cocción alcalina en agua con cal, es por mucho la operación culinaria y tecnológica más sofisticada de todas y un rasgo distintivo en la cocina mesoamericana que sobrevive hasta nuestros días. No solo es la base de al menos la mitad de las preparaciones culinarias (Echeverría y Arroyo, 2000), también ha sido modernizada y adoptada por la industria de la masa y la tortilla, uno de los sectores de mayor relevancia económica en el país (SIAP, 2007). A pesar de que este proceso conduce a pérdidas de algunas vitaminas y aminoácidos por el tratamiento térmico alcalino (Bressani, 2008), la nixtamalización también in- duce otros cambios que desde el punto de vista nutrimental son positivos, sobre todo en lo referente a la biodisponibilidad de nutrimentos. Se ha reportado un aumento significativo en el contenido de calcio (del orden de 13 veces), el cual es biodisponible prácticamente en su totalidad (Bressani, 2008). También es mayor la cantidad de fibra dietaria soluble e incrementa la biodisponibilidad de la mayoría de los aminoácidos indispensables, lo que aumenta sensiblemente el valor biológico de la proteína (Paredes-López et al., 2009). Aunque se reportan pérdidas de niacina (que no se halla disponible en el maíz) de hasta $22 \%$ después del tratamiento alcalino (Bressani, 2008), las moléculas que quedan intactas son liberadas como ácido nicotínico para su aprovechamiento, lo que destruye el efecto pelagrógeno 
que tienen las dietas ricas en maíz crudo o tostado (Paredes-López et al., 2009). La nixtamalización también puede favorecer la formación de almidón resistente (Paredes-López et al., 2009), el cual al no ser digerido se comporta de forma similar a la fibra soluble, con los beneficios para la salud que esto conlleva. Adicionalmente, se ha reportado la degradación de aflatoxinas durante la nixtamalización y la elaboración de tortillas (Méndez-Albores et al., 2004).

Los beneficios nutrimentales así como los extraordinarios cambios funcionales y sensoriales que resultan de las complejas operaciones culinarias a las que es sometido el maíz, hacen evidente el valor que tiene el conocimiento tradicional y la necesidad de sustentarlo científicamente.

\section{CARACTERIZACIÓN DE MAÍCES NATIVOS Y ASPECTOS DE CALIDAD}

En términos científicos, los usos culinarios y especiales de las razas nativas de maíz quedan determinados por las características fisicoquímicas del grano. El conjunto de pruebas para la evaluación de calidad incluye la determinación de la composición química, caracteres biofísicos y propiedades microestructurales de los granos, así como la evaluación de las propiedades térmicas del almidón (Narváez-González et al., 2007). Las características de mayor definición en el grano respecto al uso alimentario son la dureza (índice de flotación), el tamaño y la gravedad específica (peso hectolítrico) así como la capacidad de absorción de agua (Mauricio-Sánchez et al., 2004). En relación con esto, en el Cuadro 3 se especifican algunos de los criterios de calidad más sobresalientes reportados para usos culinarios comunes.

Durante mucho tiempo la evaluación de la calidad de los maíces nativos se centró en la capacidad nixtamalera y tortillera, y en la literatura científica hay varios trabajos interesantes (Antuna-Grijalva et al., 2008; Mauricio-Sánchez et al., 2004; Rangel-Meza et al., 2004; Salinas-Moreno et al., 2010; Vázquez-Carrillo et al., 2003; Vázquez-Carrillo et al., 2010 y Vázquez-Carrillo et al., 2011a).

Recientemente se han realizado valiosas investigaciones con el fin de evaluar otras cualidades en las razas nativas. Sobre calidad pozolera, Vázquez-Carrillo et al. (2011b) centraron su atención en la raza Blando de Sonora, mientras

Cuadro 3: Criterios de calidad reportados para usos comunes del maíz.

\begin{tabular}{lll}
\hline Uso & Criterios de calidad & Razas destacadas \\
\hline
\end{tabular}

Granos duros o semiduros. Valores intermedios-altos de peso de mil granos (PMG). Valores intermedios-bajos de gravedad específica. Valores altos de capacidad de absorción de agua, alto rendimiento de tortilla, baja pérdida de peso y baja resistencia al corte.

Granos muy suaves y harinosos (con alto porcentaje de endospermo suave), Atoles lo que contribuye al desarrollo de viscosidad. Valores altos de peso de mil granos (PMG). Valores bajos de gravedad específica. Valores altos de capacidad de absorción de agua.

Granos pequeños, cristalinos, muy duros y con altos valores de gravedad Palomitas específica. Valores bajos de capacidad de absorción de agua. Valores altos de volumen de expansión. Tiempos cortos de reventado.

Pinoles

Granos de baja dureza. Valores intermedios de gravedad específica y peso de mil granos (PMG). Valores bajos de capacidad de absorción de agua.

Granos suaves y harinosos (con alto porcentaje de endospermo suave), de preferencia grandes. Valores altos de peso de mil granos (PMG). Valores Pozoles bajos de gravedad específica. Valores altos de capacidad de absorción de agua. Tiempos cortos de cocción para el reventado del grano. Valores altos de capacidad de expansión.
Pepitilla, Azul, Tuxpeño, Tabloncillo, Chalqueño, Olotillo, Celaya, Onaveño, Cristalino de Chihuahua.

Cacahuacintle, Blando de Sonora, Bofo, Harinoso de Ocho, Elotes Occidentales, Ancho.

Reventador, Palomero Toluqueño, Arrocillo.

Chapalote, Elotes Cónicos, Chalqueño, Onaveño, Cacahuacintle, Dulce de Jalisco, Dulcillo del Noroeste, Bofo.

Cacahuacintle, Ancho, Blando de Sonora, Elotes Occidentales.

Elaboración propia a partir de Vázquez-Carrillo et al. (2011b), CONABIO (2010) y Mauricio-Sánchez et al. (2004). 
que Bonifacio et al. (2005) lo hicieron con la raza Cacahuacintle. Vázquez-Carrillo et al. (2011b) también investigaron la aptitud de algunas razas nativas de la región serrana de Sonora para ser usadas en la elaboración de preparaciones culinarias tradicionales, además de la tortilla.

Con respecto al contenido de nutrientes y fitoquímicos de interés en las variedades nativas, existen varios trabajos. Se ha determinado la presencia de componentes bioactivos en maíces pigmentados (López-Martínez et al., 2009; Salinas-Moreno et al., 2012; Salinas-Moreno et al., 2013) y en sus productos (Aguayo-Rojas et al., 2012; Mora-Rochín et al., 2010 ). Otros investigadores se han enfocado en el contenido de aceite de algunas variedades nativas (CoutiñoEstrada et al., 2008; Torres-Morales et al., 2010) y también se ha estudiado el contenido de azúcares en maíces eloteros (Coutiño-Estrada et al., 2010). Vidal-Martínez et al. (2008) determinaron la calidad proteínica de colectas de razas nativas de Nayarit, mientras que Vera et al. (2012) lo hicieron con variedades nativas mixtecas.

\section{Impulso al uso de maíces nativos, Una estrategia para su conservación}

La conservación del germoplasma nativo depende fundamentalmente de la protección que se otorgue a los agricultores en pequeña escala a través de subsidios, asesoría técnica, y con programas de desarrollo rural bien planeados y adaptados a las condiciones reales del medio (Kato et al., 2009). Adicionalmente, la revalorización de los usos tradicionales y el impulso estratégico de usos novedosos, pueden contribuir notablemente a la conservación in situ de los maíces nativos. Las acciones que se propongan para potenciar el uso de variedades nativas locales deben considerar las barreras propias de la composición y características del grano, las barreras del manejo poscosecha y las de carácter organizacional y económicas (Hellin et al., 2010), además de los factores socioculturales. Al respecto, los estudios de cadenas de valor son fundamentales.

Con base en estas consideraciones, se plantean las siguientes estrategias para potenciar la demanda de las diversas razas nativas de maíz:

- Identificación de propiedades nutrimentales que confieran calidad superior a las variedades nativas, con énfasis en aquellas poblaciones que se caracterizan por una alta calidad proteínica, mayor contenido de aceite o por la presencia de componentes bioactivos. Ello permitirá, además, aplicar precios diferenciales por calidad.

- Desarrollo de sistemas de conservación apropiados para productos tradicionales que permitan potenciar su venta en mercados de exportación, por ejemplo, tamalitos congelados.

- Desarrollo de productos novedosos con base en las características fisicoquímicas que los hacen aptos para un uso particular, por ejemplo, nuevas botanas (extrudidas o no) de colores novedosos.

- Extracción de pigmentos con aplicaciones en diversas industrias, alimentarias y no alimentarias.

- Extracción de fracciones específicas del grano que sean de interés industrial; por ejemplo: fibras para aplicaciones alimentarias, zeínas para aplicaciones como empaques comestibles, péptidos bioactivos, almidones resistentes a la digestión, etc.

- Realización de ferias de diversidad para muestra e intercambio de semillas, así como exposición de usos y preparaciones culinarias tradicionales. Estas son actividades que contribuirán a mantener la siembra de maíces nativos en toda su diversidad, y que ya han dado resultados interesantes como lo mostraron Aragón-Cuevas et al. (2011).

- Desarrollo de marcas colectivas, alternativa que con frecuencia ha mostrado ser muy eficiente en la conservación in situ y resguardo de los mercados tradicionales, además de convertirse en un motor del mejoramiento participativo (R. Ortega-Paczka; Com. pers. ${ }^{2}$ ). Algunas marcas colectivas ya han sido propuestas por AragónCuevas et al. (2006): "Totopos del Istmo", elaborados con el maíz Zapalote Chico, o bien, "Tlayudas del Valle", tortillas elaboradas con maíz Bolita.

- Adquisición de certificados de calidad o en los que se resalte alguna cualidad de interés para ciertos consumidores en particular, por ejemplo: "producto orgánico elaborado con maíces nativos".

\section{CONCLUSIONES}

Los maíces nativos, además de ser parte del patrimonio biocultural que México ha regalado al mundo, son el sustento de miles de familias en zonas rurales, por lo que su protección y conservación resulta fundamental. Se han identificado una serie de ventajas para las variedades locales en las pequeñas unidades de producción, entre las que destaca la posibilidad de hacer un mejor manejo del riesgo agrícola, lo que proporciona mayores garantías en la producción alimentos en cantidad suficiente.

Por otro lado, están los usos especiales de las razas nativas de maíz en la alimentación, las cuales presentan características únicas que las hacen insustituibles para preparar una gran variedad de platillos tradicionales. Es así como la cocina tradicional mexicana, considerada Patrimonio Cultural Inmaterial de la Humanidad, requiere obligatoriamente del

${ }^{2}$ Rafael Ortega Paczka. Universidad Autónoma Chapingo. Noviembre de 2010: Aportes para diseñar un modelo de conservación de los maíces criollos en México. 
uso de variedades nativas por la calidad que confieren a las preparaciones culinarias.

Dada la clara relación que en muchos casos existe entre raza y uso especial, recientemente se han realizado importantes esfuerzos orientados a la definición de los parámetros de calidad que determinan el uso final, lo que ha requerido de la caracterización fisicoquímica de los granos de diversas razas nativas de maíz. Asimismo, se ha generado información científica valiosa que ha permitido identificar usos novedosos. Aunque todavía hay vacíos en el campo de investigación, todas estas acciones proporcionan el sustento científico que requiere el impulso estratégico de la siembra de maíces nativos, lo que puede contribuir notablemente a su conservación in situ.

\section{BIBLIOGRAFÍA}

Aguayo-Rojas J, S Mora-Rochín, E O Cuevas-Rodríguez, S O Serna-Saldivar, J A Gutiérrez-Uribe, C Reyes-Moreno, J Milán-Carrillo (2012) Phytochemicals and antioxidant capacity of tortillas obtained after Lime-Cooking Extrusion Process of whole pigmented Mexican Maize. Plant Foods Human Nutr. 67:178-185.

Antuna-Grijalva O, S A Rodríguez-Herrera, G Arámbula-Villa, A Palomo-Gil, E Gutiérrez-Arias, A Espinosa-Banda, E F Navarro-Orona, E Andrio-Enríquez (2008) Calidad nixtamalera y tortillera en maíces criollos de México. Rev. Fitotec. Mex. 31:23-27.

Aragón-Cuevas F, F H Castro-García, J M Cabrera Toledo, L OsorioAlcalá (2011) Bancos Comunitarios de Semillas para Conservar in situ la Diversidad Vegetal. Publicación especial No. 9. Instituto Nacional de Investigaciones Forestales, Agrícolas y Pecuarias, Centro de Investigación Regional Pacífico Sur, Campo Experimental Valles Centrales de Oaxaca. Santo Domingo Barrio Bajo, Etla, Oaxaca, México. 79 p.

Aragón-Cuevas F, S Taba, J M Hernández, J D Figueroa, V Serrano (2006) Actualización de la Información sobre los Maíces Criollos de Oaxaca. Instituto Nacional de Investigaciones Forestales Agrícolas y Pecuarias, Informe final SNIB-CONABIO proyecto No. CS002 México D. F. 119 p.

Beas F R, G Loarca, S H Guzmán, M G Rodríguez, N L Vasco, F Guevara (2011) Potencial nutracéutico de componentes bioactivos presentes en huitlacoche de la zona centro de México. Rev. Mex. Cien. Farm. 42:36-44.

Bellon M R, D Hodson, J Hellin (2011) Assessing the vulnerability of traditional maize seed systems in Mexico to climate change. Proc. Nat. Acad. Sci. USA. 108:13432-13437.

Bonifacio-Vázquez E I, Y Salinas-Moreno, A Ramos Rodríguez, A Carrillo-Ocampo (2005) Calidad pozolera en colectas de maíz cacahuacintle. Rev. Fitotec. Mex. 28:253-260.

Bourges-Rodríguez H (2004) Abasto y consumo de alimentos: una perspectiva nutriológica. In: El Desarrollo Agrícola y Rural del Tercer Mundo en el Contexto de la Mundialización. M C Del Valle-Rivera (ed). UNAM-IIES-Plaza y Valdés. D.F, México. pp:433-451.

Bressani R (2008) Cambios nutrimentales en el maíz inducidos por el proceso de nixtamalización. In: Nixtamalización del Maíz a la Tortilla. Aspectos Nutrimentales y Toxicológicos. M E Rodríguez-García, S O Serna-Saldívar, F Sánchez-Sinencio (eds). Universidad Autónoma de Querétaro. Querétaro, México. pp:19-80.

CONABIO, Comisión Nacional para el Conocimiento y Uso de la Biodiversidad (2011) Proyecto global "Recopilación, generación, actualización y análisis de información acerca de la diversidad genética de maíces y sus parientes silvestres en México". Disponible en: http://www.biodiversidad.gob.mx/genes/proyectoMaices.html. (Mayo 2013).
CONEVAL, Consejo Nacional de Evaluación de la Política de Desarrollo Social (2012) Construcción de las Líneas de Bienestar. Documento metodológico. Disponible en: http://www.coneval. gob.mx/Informes/Coordinacion/INFORMES_Y_PUBLICACIONES_PDF/Construccion_lineas_bienestar.pdf.

Coutiño-Estrada B, A Ortega-Corona, V A Vidal-Martínez, G SánchezGrajalez, S I García-Acuña (2008) Selección recurrente para incrementar el contenido de aceite en maíz comiteco. Rev. Fitotec. Mex. 31:5-8.

Coutiño-Estrada B, V A Vidal-Martínez, B Cruz-Gómez, C CruzVázquez (2010) Aptitud combinatoria general y específica del contenido de azúcares en maíces criollos eloteros. Rev. Fitotec. Mex. 33:57-61.

Cravioto R O, G Massieu, J Guzmán (1955) Investigaciones bromatológicas en alimentos mexicanos. Bol. Of. Sanit. Panam. 30:26-33.

Echeverría M E, L E Arroyo (2000) Recetario del Maíz. Cocina Indígena y Popular. Consejo Nacional para las Culturas y las Artes (CONACULTA). D.F., México. 441 p.

FAOSTAT (2009) Food Supply. Crops Primary Equivalent. Data Base. Consultado el 7 de mayo de 3013. Disponible en: http://faostat3.fao.org/home/index.html\#DOWNLOAD (Mayo 2013).

Gálvez-Mariscal A, H Bourges-Rodríguez (2012) La alimentación en la Ciudad de México. In: Los Riesgos para la Salud en la Vida de una Megametrópoli. Memoria I. Congreso Internacional. Facultad de Medicina UNAM, 25 y 26 de agosto. Universidad Nacional Autónoma de México. D.F., México. pp:366-403.

García-Urigüen P (2012) La Alimentación de los Mexicanos. Cambios Sociales y Económicos, y su Impacto en los Hábitos Alimenticios. Cámara Nacional de la Industria de la Transformación (CANACINTRA) D.F., México. $162 \mathrm{p}$

Guillén-Pérez, L A, C Sánchez-Quintanar, S Mercado-Domenech, H Navarro-Garza (2002) Análisis de atribución causal en el uso de semilla criolla y semilla mejorada de maíz. Agrociencia 36:377-387.

Hellin J, A Keleman, G Atlin (2010) Smallholder Farmers and Maize in Mexico: A Value-Chain Approach to Improved Targeting of Crop-Breeding Programs. J. New Seeds 11:262-280.

INEGI, Instituto Nacional de Estadística y Geografía (2010) Encuesta Nacional de Ingresos y Gastos de los Hogares (ENIGH). Disponible en: http://www.inegi.org.mx/est/contenidos/Proyectos/Encuestas/Hogares/regulares/Enigh/ (Mayo 2013).

Juárez M M, S Ruiloba de León, G Chávez, C Hernández, L Villa (2011) Huitlacoche (corn smut), caused by the phytopathogenic fungus Ustilago maydis, as a functional food. Rev. Iberoam. Micol. 25:69-73.

Kato T A, C Mapes, L M Mera, J A Serratos, R A Bye (2009) Origen y Diversificación del Maíz: Una Revisión Analítica. Universidad Nacional Autónoma de México, Comisión Nacional para el Uso y Conocimiento de la Biodiversidad. Editorial Impresora Apolo, S.A. de C.V. D.F., México. 116 p.

Keleman A, J Hellin (2009) Specialty maize varieties in Mexico: A case study in market-driven agro-biodiversity conservation. J. Lat. Am. Geogr. 8:147-174.

Lazos E, M Chauvet (2011) Análisis del contexto social y biocultural de las colectas de maíces nativos en México. Proyecto global de maíces nativos. Informe de Gestión. CONABIO. Disponible en: http://www.biodiversidad.gob.mx/genes/pdf/proyecto/ Anexo9_Analisis_Especialistas/Lazos\%20y\%20Chauvet\%20 2011.pdf (mayo 2013).

López M (2007) Antiguas Representaciones del Maíz. CONACULTA/Archivo General de la Nación. D.F., México. 89 p.

López-Martínez L X, R M Olart-Ros, G Valerio-Alfaro, C H Lee, K L Parkin, H S García (2009) Antioxidants activity, phenolic compounds and anthocyanins content of eighteen strains of Mexican maize. LWT - Food Sci. Technol. 42:1187-1192.

Mauricio-Sánchez R A, J D Figueroa-Cárdenas, S Taba, M L Reyes-Vega, F Rincón-Sánchez, A Mendoza-Galván (2004) Caracterización de accesiones de maíz por calidad de grano y tortilla. Rev. Fitotec. Mex.27:213-222.

Mera-Ovando L M (2009) Aspectos socioeconómicos y culturales. In: Origen y Diversificación del Maíz: Una Revisión Analítica. T A Kato, C Mapes, L M Mera, J A Serratos, R A Bye (eds). Universidad Nacional Autónoma de México, Comisión Nacional para 
el Uso y Conocimiento de la Biodiversidad. Editorial Impresora Apolo, S.A. de C.V. D.F., México. pp:33-42.

Mera-Ovando L M, C Mapes-Sánchez (2009) El maíz. Aspectos biológicos. In: Origen y Diversificación del Maíz: Una Revisión Analítica. T A Kato, C Mapes, L M Mera, J A Serratos, R A Bye (eds). Universidad Nacional Autónoma de México, Comisión Nacional para el Uso y Conocimiento de la Biodiversidad. Editorial Impresora Apolo, S.A. de C.V. D.F., México. pp:19-32.

Méndez-Albores J A, G Arámbula-Villa, M G Loarca-Piña, J GonzálezHernández, E. Castaño-Tostado, E Moreno-Martínez (2004) Aflatoxins fate during the nixtamalization of contaminated maize by two tortilla-making processes. J. Stored Prod. Res. 40:87-94.

Miranda-Colín S (2000) Mejoramiento genético del maíz en la época prehispánica. Agric. Téc. Méx. 26:3-15.

Mora-Rochín S, J A Gutiérrez-Uribe, S O Serna-Saldívar, P SánchezPeña, C Reyes-Moreno, J Milán-Carrillo (2010) Phenolic content and antioxidant activity of tortillas produced from pigmented corns processed by conventional nixtamalization or extrusion cooking. J. Cereal Sci. 52:502-508.

Narváez-González E D, J D Figueroa-Cárdenas, S Taba (2007) Aspectos microestructurales y posibles usos del maíz de acuerdo con su origen geográfico. Rev. Fitotec. Mex. 30:321-325.

Ortega-Corona A, M J Guerrero-Herrera, O Cota-Agramont, R E Preciado-Ortíz (2011) Situación actual de los maíces nativos y sus parientes silvestres en México. In: Amplitud, Mejoramiento, Usos y Riesgos de la Diversidad Genética de Maíz en México. R E Preciado-Ortíz, S Montes-Hernández (eds). Sociedad Mexicana de Fitogenética, A.C. Chapingo, Estado de México, México. pp:15-41.

Ortega-Paczka R (2003) La diversidad del maíz en México. In: Sin Maíz No Hay País. G Esteva, C Marielle (eds). Culturas Populares de México. D.F., México. pp:123-154.

Paredes-López O, F Guevara-Lara, L A Bello-Pérez (2009) La nixtamalización y el valor nutritivo del maíz. Ciencias 92-93:60-70.

Rangel-Meza E, A Muñoz-Orozco, M G Vázquez-Carrillo, J CuevasSánchez, J Merino-Castillo, J S Miranda-Colín (2004) Nixtamalización, elaboración y calidad de tortilla de maíces de Ecatlán, Puebla, México. Agrociencia 38:53-61.

Salinas-Moreno Y, F Aragón-Cuevas, C Ybarra-Moncada, J AguilarVillarreal, B Altunar- López, E Sosa-Montes (2013) Caracterización física y composición química de razas de maíz de grano azul/morado de las regiones tropicales y subtropicales de Oaxaca. Rev. Fitotec. Mex. 36:23-31.

Salinas-Moreno Y, F J Cruz-Chávez, S A Díaz-Ortiz, F Castillo-González (2012) Granos de maíces pigmentados de Chiapas, características físicas, contenido de antocianinas y valor nutracéutico. Rev. Fitotec. Mex. 35:33-41.

Salinas-Moreno Y, N O Gómez-Montiel, J E Cervantes-Martínez, M Sierra-Macías, A Palafox-Caballero, E Betanzos-Mendoza, B Coutiño-Estrada (2010) Calidad nixtamalera y tortillera en maíces del trópico húmedo y sub-húmedo de México. Rev. Mex. Cien. Agríc. 1:509-523.

Sánchez J J, M M Goodman, C W Stuber (2000) Isozymatic and morphological diversity in the races of maize of Mexico. Econ.c Bot- 54:43-59.

Serna-Saldívar S O, C A Amaya-Guerra (2008) El papel de la tortilla nixtamalizada en la nutrición y la alimentación. In: Nixtamalización del Maíz a la Tortilla. Aspectos Nutrimentales y Toxicológicos. M E Rodríguez-García, S O Serna-Saldívar, F Sánchez-Sinencio (eds). Universidad Autónoma de Querétaro. Querétaro, México. pp:105-151.

SIAP, Servicio de Información Agroalimentaria y Pesquera (2007) Situación Actual y Perspectivas del Maíz en México 1996 - 2012. Secretaría de Agricultura, Ganadería, Desarrollo Rural, Pesca y Alimentación (SAGARPA). México, D.F. 208 p.

SIAP, Servicio de Información Agroalimentaria y Pesquera (2011) Cierre de la producción agrícola por cultivo. Disponi- ble en: http://www.siap.gob.mx/index.php?option=com_ wrapper\&view=wrapper\&Itemid=215 (Mayo 2013).

SINAREFI, Sistema Nacional de Recursos Fitogenéticos para la Alimentación y la Agricultura (2012) Políticas Públicas Red Maíz/Custodios y Razas/Fichas Técnicas. Disponible en: http:// www.sinarefi.org.mx/basicos_maiz.html (Octubre 2012).

Taube K (1989) The maize tamale in Classic maya diet, epigraphy and art. Amer. Antiquity 54:13-51.

Toledo-Manzur V M, N Barrera-Bassols (2008) La Memoria Biocultural. La Importancia Ecológica de las Sabidurías Tradicionales. Icaria Editorial, S.A. Barcelona, España. 233 p.

Torres-Morales B, B Coutiño-Estrada, A Muñoz-Orozco, A SantacruzVarela, A Mejía-Contreras, S O Serna-Saldivar, S GarcíaLara, N Palacios-Rojas (2010) Selección para contenido de aceite en el grano de variedades de maíz de la raza comiteco de Chiapas, México. Agrociencia. 44:679-689.

Turiján-Altamirano T, M A Damián-Huato, B Ramírez-Valverde, J P Juárez-Sánchez, N Estrella-Chulím (2012) Manejo tradicional e innovación tecnológica en cultivo de maíz en San José Chiapa, Puebla. Rev. Mex. Cien. Agríc. 3:1085-1110.

Turrent-Fernández A, J I Cortés-Flores, A Espinosa-Calderón, H Mejía-Andrade, J A Serratos-Hernández (2010) ¿Es ventajosa para México la tecnología actual de maíz transgénico? Rev. Mex. Cien. Agríc. 1:631-646.

Turrent-Fernández A, T A Wise, E Garvey (2012) Factibilidad de alcanzar el potencial productivo de maíz de México. Mex. Rural Develop. Res. Rep. 24:1-36.

UNESCO, Organización de las Naciones Unidas para la Educación, la Ciencia y la Cultura (2010) La Lista Representativa del Patrimonio Cultural Inmaterial de la UNESCO se enriquece con 46 nuevos elementos, 16 de noviembre. Disponible en: http://www.unesco.org/new/es/media-services/single-view/ news/forty_six_new_elements_added_to_representative_ list_of_the_intangible_cultural_heritage/\#.UmVtGnCP8rw (Mayo 2013).

Valadez Azúa R, A Moreno Fuentes, G Gómez Álvarez (2011) Cujtlacochi. El Cuitlacoche. IIA-UNAM. D.F., México. 138 p.

Vázquez-Carrillo M G, S García-Lara, Y Salinas-Moreno, D J Bergvinson, N Palacios-Rojas (2011a) Grain and tortilla quality in landraces and improved maize grown in the highlands of Mexico. Plant Foods Human Nutr. 66:203-208.

Vázquez-Carrillo M G, L Guzmán-Báez, J L Andrés-García, F MárquezSánchez, Castillo-Merino J (2003) Calidad de grano y tortillas de maíces criollos y sus retrocruzas. Rev. Fitotec. Mex. 26:231238.

Vázquez-Carrillo M G, A Ortega-Corona, M J Guerrro-Herrera, B Coutiño-Estrada (2011b) Evaluación bioquímica e industrial de razas nativas de maíz de la región serrana de Sonora. In: Amplitud, Mejoramiento, Usos y Riesgos de la Diversidad Genética de Maíz en México. R E Preciado-Ortíz, S Montes-Hernández (eds). Sociedad Mexicana de Fitogenética, A.C. Chapingo, Estado de México, México. pp:97-142.

Vázquez-Carrillo M G, J P Pérez-Camarillo, J M Hernández-Casillas, M L Marrufo-Diaz, E Martínez-Ruiz (2010) Calidad de grano y de tortillas de maíces criollos del altiplano y valle del mezquital, México. Rev. Fitotec. Mex. 33:49-56.

Vera-Guzmán A M, J L Chávez-Servia, J C Carrillo-Rodríguez (2012) Proteína, lisina y triptófano en poblaciones nativas de maíz mixteco. Rev. Fitotec. Mex. 35:7-13.

Vidal-Martínez V A, M G Vázquez-Carrillo, B Coutiño-Estrada, A Ortega-Corona, J L Ramírez-Díaz, R Valdivia-Bernal, M J Guerrero-Herrera, F J Caro-Velarde, O Cota-Agramont (2008) Calidad proteínica en colectas de maíces criollos de la sierra de Nayarit, México. Rev. Fitotec. Mex. 31:15-21.

Wacher C, A Cañas, P E Cook, E Barzana, J D Owens (1993) Sources of microorganisms in pozol, traditional Mexican fermented maize dough. World J. Microbiol. Biotechnol. 9:269-274. 